\title{
Description du mâle d'Orphulella trypha Otte, 1979, des Antilles
} françaises (Orth., Acrididae)

Jacques Bonfils, Antoine Foucart

\section{Citer ce document / Cite this document :}

Bonfils Jacques, Foucart Antoine. Description du mâle d'Orphulella trypha Otte, 1979, des Antilles françaises (Orth., Acrididae). In: Bulletin de la Société entomologique de France, volume 101 (4), octobre 1996. pp. 379-380; https://www.persee.fr/doc/bsef_0037-928x_1996_num_101_4_17266

\section{Ressources associées :}

Orphulella trypha

Fichier pdf généré le 24/09/2019 
and Linognathoides (Polyplacidae: Anoplura). Journal of Medical Entomology, 19 : 615-627.

KIM K.C. \& EMERSON K.C., 1968. - New records and nymphal stages of the anoplura from central and east africa, with description of a new Hoplopleura species. Revue de Zoologie et de Botanique Africaines, 78 : 5-45.

1973. - Anoplura of tropical west africa with descriptions of new species and nymphal stages. Revue de Zoologie et de Botanique Africaines, 87 : 426-455.

KIM K.C. \& LudWIG H.W., 1978. - The family classification of the Anoplura. Systematic entomology, 3 : 249-284.

KuHN H.J. \& Ludwig H.W., 1964. - Pedicinus patas (Fahrenholz) (Anoplura) and other lice of the cercopithecini. Annals and Magazin of Natural History, (13) 7: 513-522.

1965. - Anoplura liberianischer Nagar. Seckenbergiana biologica, 46 : 233-244.

1967. - Die affenläuse der Gattung Pedicinus. Zeitschrift für Zoologische Systematik und Evolutionsforschung, 5 : 144-297.

LEDGER J.A., 1980. - The arthropod parasites of vertebrates in Africa south of the Sahara. Volume IV. Phthiraptera (Insecta). Publication of the South African Institute for Medical Research $\mathrm{n}^{\circ}$ 56, $327 \mathrm{pp}$.

Musser G.G. \& CARLETON M.D., 1993. - Family Muridae in Wilson D.E. and Reeder D.M. (eds.), Mammals species of the world, a taxonomic and geographic reference, 2nd ed, pp. $501-$ 755. Washington, D.C. : Smithsonian Inst. Press.

PAJOT F.-X., 1967. - Anoploures de rongeurs et d'insectivores de la République centrafricaine. Cahiers O.R.S.T.O.M., série Entomologie médicale, 5 : 107-114.

- 1968. - Lemurphthirus vincenti sp. n. (Insecta, Anoplura) du Congo (Brazzaville) parasite du galago de Demidoff (Lémurien). Cahiers O.R.S.T.O.M., série Entomologie médicale, 6 : 191-196.

Pajot F.-X. \& Germain M., 1971. - Note sur un nouveau cas de phorésie chez les insectes. Transport de Linognathus breviceps (Piaget) (Anoplura : Linognathidae) par des Eretmapodites du groupe chrysogaster (Diptera, Culicidae). Bulletin de la Société entomologique de France, 76 : 5-6.

Paulian R. \& PAJOT F.-X., 1966. - Anoploures de la République centrafricaine et du Congo (Brazzaville). Bulletin de la Société entomologique de France, $71: 40-51$.

Rosevear D.R., 1969. - The rodents of West Africa. London: Trustees of the British Museum (Natural History), 677, $1604 \mathrm{pp.}$

WeRnECK F.L., 1953. - Contribuçao ao conhecimentos dos anopluros IV. Revista Brasileira Biologia, 13 : 53-64.

WORTH C.B. \& PATERSON H.E., 1960. - Phoresy of sucking lice (Siphunculata : Linognathidae) by a mosquito (Diptera : Culicidae). Journal of Entomological Society of Southern Africa, 23 : 228-230.

\section{Jacques BONFILS et Antoine FouCART. - Description du mâle d'Orphulella trypha Otte, 1979, des Antilles françaises (Orth., Acrididae)}

Parmi les six espèces de Gomphocerinae du genre Orphulella de la région Caraïbe, trois y sont microptères : Orphulella descisa (Walker, 1870), O. nesicos Otte, 1979, et $O$. trypha Otte, 1979. Cette dernière a été décrite de la partie hollandaise de l'île de Saint-Martin, sur un exemplaire femelle (OTTE, 1979: 67 et 84.), l'holotype est déposé à l'Académie des Sciences naturelles de Philadelphie (Etats-Unis). Nous donnons ici la description du mâle à partir du matériel provenant des récoltes d'Orthoptéroïdes effectuées par J. Bonfils aux Antilles de 1960 à 1968. en particulier dans l'île de Saint-Martin ainsi que dans l'île de Saint-Barthélémy.

Matériel étudié déposé en partie (dont le néallotype) au Muséum national d'Histoire naturelle de Paris (MNHN) et à l'École nationale supérieure agronomique de Montpellier (ENSA-M) :

- Ile de Saint-Martin: Marigot, Baie Nettlé, $1 \delta^{\star}$ néallotype, $11 \delta^{\star}, 4 \%$ (MNHN), $50^{\circ}, 4 \%$ (ENSA-M) 10.VI. 1966, pelouse littorale sur sable blanc; Philipsburg, 30, 1 ( (MNHN) 30.III.1963; Cul-de-Sac, 30 (MNHN), $30^{\circ}$ (ENSA-M), 31.III.1963 ; Baie-aux-Cailles, $40^{\circ}, 2 \circ$ (MNHN) 30.III.1963 (J. Bonfils leg.). 
- Ile de Saint-Barthélémy: Quartier de Lorient, lande littorale pacagée, $15 \delta^{\star}$ (MNHN) et $3 \delta^{\star}$ (ENSA-M), $17 \uparrow(\mathrm{MNHN})$ et $3 \%$ (ENSA-M), 10.III.1963; Quartier des Salines, lande littorales, $10 \hat{0}, 2 \uparrow$ (MNHN), 20.IX.1966 ( tous J. Bonfils leg.).

Description: Le mâle diffère de la femelle par la taille, presque deux fois plus petite (fig. 1). Taille du néallotype: $10.5 \mathrm{~mm}$, forme robuste, tégument lisse. Fastigium du vertex à face dorsale subtriangulaire, les carinules latérales peu saillantes, le disque subplan à l'avant, légèrement sillonné à la base. Côte frontale étroite et sillonnée au-dessus de l'ocelle médian, plus large et subplane au-dessous, linéaire juste au-dessous de l'angle fastigio-frontal ; fovéoles temporales subobsolètes. Yeux grands et subglobuleux. Antennes filiformes de 16 articles, plus courtes que la tête et le pronotum réunis. Pronotum à bord antérieur un peu arqué, le bord postérieur à angle concave; la carène médiane nette, lcs carènes latérales convergentes-divergentes sont incurvées dans la prozone, les lobes latéraux un peu moins hauts que longs. Élytres lobiformes latéraux, largement distants dorsalement, pas plus longs que le pronotum, atteignant le bord postérieur $\mathrm{du} 1^{\mathrm{e}}$ tergite abdominal, l'extrémité arrondie, la nervation saillante. Ailes vestigiales moitié moins longues que les élytres. Tibias postérieurs armés de huit épines sur les bords internes et externes. Intérieur des fémurs postérieurs dépourvu de peigne stridulatoire. Bord postérieur du dernier tergite abdominal à furcula bien nette; plaque sus-anale aussi longue que large à la base, les bords latéraux légèrement incurvés à angulation subapicale ; cerques un peu plus longs que larges à la base. Teinte générale brun jaunâtre clair ; face claire. Taches brun foncé dorso-latérales alignées du bord postérieur des yeux jusqu'à l'extrémité de l'abdomen. Une tache (peu prononcée chez le néallotype) brun foncé en position médiane sur la face externe et la face supérieure des fémurs postérieurs. Pronotum à carènes latérales claires ; lobes latéraux à plages brun foncé plus ou moins étendues et confluentes selon les exemplaires. Pleures thoraciques plus ou moins marqués de noir.

Deux types de colorations, brun clair et vert, s'observent dans les séries récoltées dans les mêmes stations. Ce dichroïsme est moins fréquent chez les mâles.

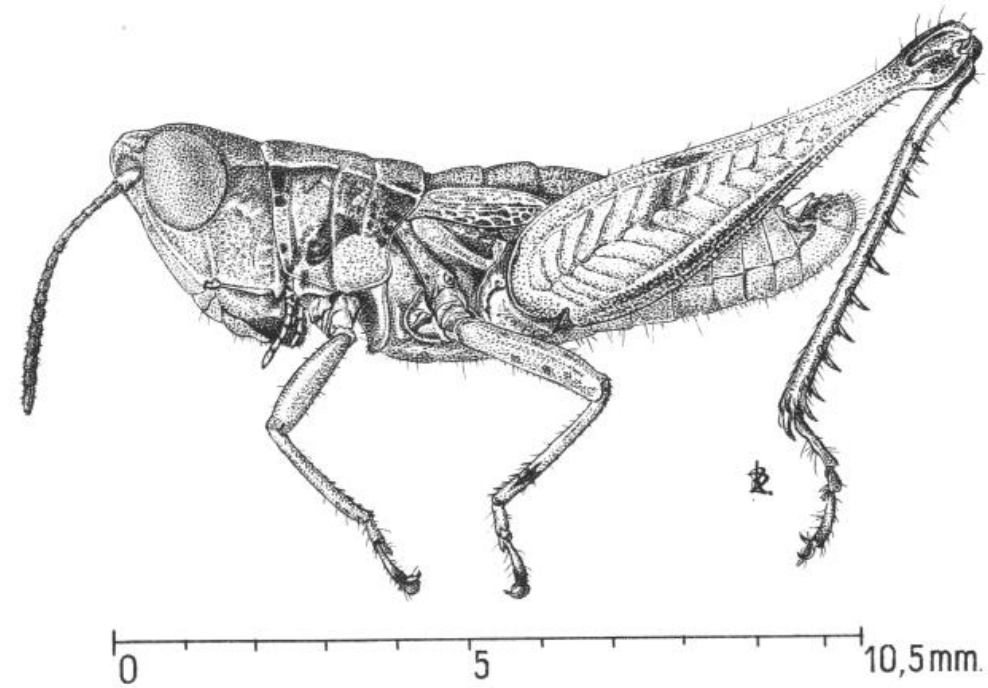

Fig. 1 : Orphulella trypha Otte, 1979, ơ néallotype, île de St-Martin (illustration de R. Préchac).

\section{AUTEUR CITÉ}

OTTE D., 1979. - Revision of the Grasshopper Tribe Orphulellini (Gomphocerinae: Acrididae). Proceedings of the Academy of Natural Sciences of Philadelphia, 131 (10) : 52-88.

1981. - The north American Grasshoppers, volume I Acrididae (Gomphocerinae and Acridinae). Cambridge, Massachusetts: Harvard University Press, 275 pp.

(J. B. : 10 rue des Bouvreuils, Aiguefonde, $F-81200$ Mazamet; (A. F. : CIRAD-GERDAT-PRIFAS, B.P. 5035, F-34032 Montpellier) 